\title{
Model-Free Control of Finger Dynamics in Prosthetic Hand Myoelectric-based Control Systems
}

\author{
Radu-Emil PRECUP ${ }^{1 *}$, Raul-Cristian ROMAN ${ }^{1}$, Teodor-Adrian TEBAN ${ }^{1}$, \\ Adriana ALBU ${ }^{1}$, Emil M. PETRIU², Claudiu POZNA ${ }^{3,4}$ \\ ${ }^{1}$ Department of Automation and Applied Informatics, Politehnica University of Timişoara, \\ 2 Vasile Pârvan Avenue, 300223 Timișoara, Romania \\ radu.precup@aut.upt.ro (*Corresponding author),raul.roman@aut.upt.ro, \\ adrian.teban@student.upt.ro, adriana.albu@aut.upt.ro \\ ${ }^{2}$ School of Electrical Engineering and Computer Science, University of Ottawa, \\ Ottawa, ON K1N 6N5, Canada \\ petriu@uottawa.ca \\ ${ }^{3}$ Department of Informatics, Széchenyi István University, Egyetem tér 1, 9026 Győr, Hungary \\ ${ }^{4}$ Department of Automation and Information Technology, Transilvania University of Brașov, \\ 5 Mihai Viteazu Street, building no. V, 3rd floor, 500174 Brașov, Romania \\ cp@unitbv.ro
}

\begin{abstract}
This paper proposes an approach to the tuning of model-free controllers for the midcarpal joint angles, which are important finger angles that ensure the desired finger dynamics in prosthetic hand myoelectric-based control systems. The process in these control systems is characterized by fuzzy models that operate with myoelectric signals obtained from eight myoelectric sensors and past inputs and/or outputs, where the controlled outputs are five finger angles. Since the fuzzy models exhibit very good performance as shown in authors' recent papers that produced evolving fuzzy models, they are used to simulate the process behaviour. The Multi Input-Multi Output (MIMO) control system structure consists of five separate Single Input-Single Output control loops with the most simple model-free controllers represented by intelligent Proportional (iP) controllers, separately designed and tuned for each finger. Digital simulation results are included to suggestively illustrate the very good performance of the control systems with iP controllers. The MIMO control system performance is compared with that of the same system but with Proportional-Integral controllers, which are optimally tuned in a model-based manner by a metaheuristic Grey Wolf Optimizer (GWO) algorithm. The fair comparison is ensured by the optimal tuning of the free parameters of iP controllers in a model-based manner using GWO.
\end{abstract}

Keywords: Model-free control, Myoelectric-based control, Fuzzy models, Intelligent Proportional controllers, ProportionalIntegral controllers.

\section{Introduction}

As shown in (Geethanjali, 2016), myoelectricbased control system structures and algorithms are based on on-off, proportional, direct, finite state machine, pattern recognition-based, posture and regression control. The control system design and tuning is usually carried out in a model-based manner that requires accurate models of the human hand, which is essentially a Multi Input-Multi Output (MIMO) nonlinear dynamical system. The inputs of this MIMO system are represented by myoelectric signals and a part of the outputs consists of finger angles at various joints.

Some recent approaches to myoelectricbased control are briefly discussed as follows. A myoelectric-driven musculoskeletal model that decodes joint moments is included in (Sartori et al., 2018) in a control scheme for wrist movement and hand opening-closing. The human-machine interface is improved in (Kapelner et al., 2019) by decomposing recorded myoelectric signals into the underlying neural drive. Using the regression convolutional neural network suggested in (Ameri et al., 2019), an adaptive auto-regressive filter algorithm based on adaptive infinite impulse response filtering theory is proposed in (Igual et al., 2019) to learn proportional velocity control. Proportional-Integral (PI) and ProportionalIntegral-Derivative (PID) controllers are applied in (Precup et al., 2020b) and (Blana et al., 2020). Fuzzy controllers for myoelectric-based control are designed in Mamdani form in (Tabakov et al., 2016; Tabakov et al., 2018) and Takagi-SugenoKang form in (Precup et al., 2020b).

An alternative to the model-based tuning of controllers is represented by data-driven control, where few information is used on the process model considering that no process model is involved in the controller tuning. However, using input-output data from the process instead of parametric models in the controller tuning may be supported by non-parametric models of the process expressed as time responses or 
frequency domain plots. This important feature of data-driven control makes the associated techniques of great interest in myoelectric-based control systems as there is no more need to focus efforts on deriving accurate process models as experiments actually play the role of learning algorithms that automatically tune the controller parameters. Few iterations are usually used in this regard, making the controller tuning relatively simple for practitioners in various multi-disciplinary subdomains of biomedical engineering.

Some of the most successful data-driven techniques will be highlighted as follows citing both classical and fresh papers that actually apply them: Iterative Feedback Tuning (IFT) (Hjalmarsson, 2002; Jung et al., 2020), Modelfree Adaptive Control (Hou \& Wang, 2013; Yu et al., 2020), Simultaneous Perturbation Stochastic Approximation (Spall \& Cristion, 1998; Zamanipour, 2020), Correlation-based Tuning (Karimi et al., 2004; Sato, 2020), Frequency Domain Tuning (Kammer et al., 2000; da Silva Moreira et al., 2018), Iterative Regression Tuning (Halmevaara \& Hyötyniemi, 2006), and adaptive online IFT (McDaid et al., 2012). While these data-driven techniques actually carry out the iterative experiment-based update of controller parameters, non-iterative techniques are also popular as Model-Free Control (MFC) (Fliess \& Join, 2009, 2013), Virtual Reference Feedback Tuning (Campi et al., 2002; Formentin et al., 2019); Active Disturbance Rejection Control (Gao, 2006; Roman et al., 2020), data-driven predictive control (Kadali et al., 2003; Lucchini et al., 2020), unfalsified control (Safonov \& Tsao, 1997; Jiang et al., 2016) and Data-Driven Inversion Based Control (Novara et al., 2015; Galluppi et al., 2019). As shown in (Precup et al., 2020a), the approach reported in (Van Waarde et al., 2020) does not make the a priori assumption of persistency of excitation on the system input; instead, it studies equivalent conditions on the given data under which different analysis and control problems can be solved. Thus, it reveals situations in which a controller can be tuned from data even though unique system identification is impossible. A fresh review on data-driven control is conducted in (Huang \& Gao, 2020) giving useful classifications and pointing out the role of observers and estimation in control.
MFC combines the well-known and relatively easily understandable PI and PID controller structure with an intelligent term that compensates for the effects of nonlinear dynamics, disturbances or uncertain parameters. An integral local model of the process is used in this regard, but its identification is not needed. Model-free controllers represented by intelligent Proportional (iP) and intelligent Proportional-Integral (iPI) controllers are designed making use of a so-called estimated data-driven term. Some recent popular applications of MFC include treatment of acute inflammation (Bara et al., 2018), handling packet losses and latencies in industrial Internet of Things (Join et al., 2020) two rotor aerodynamic systems (Roman et al., 2016; Roman et al., 2018) and control of upper-limb exoskeletons (Wang et al., 2020). All these applications and performance proved by MFC justify to consider, as stated in (Fliess \& Join, 2020), that MFC is a new tool for Machine Learning. Moreover, this paper will prove, besides other recent applications of MFC, that it is an efficient tool for Machine Learning.

This paper is developed on the basis of the evolving Takagi-Sugeno-Kang fuzzy models derived in (Precup et al., 2018, 2019) and also used to finger control in (Precup et al., 2020b), to characterize the nonlinear finger dynamics of the human hand in the framework of myoelectricbased control of a prosthetic hand. The inputs of these models are the myoelectric signals obtained from eight sensors placed on human subject's arm, and the outputs are the flexion percentages that correspond to the midcarpal joint angles. For the sake of simplicity, the flexion percentages are next referred to also as finger angles. Using the MIMO control system structure suggested in (Precup et al., 2020b) consisting of five control loops to control the angles of each finger, this paper proposes iP controllers, which are separately designed and tuned in a modelfree manner for each finger. The MIMO control system behaviour is simulated using the best fuzzy models for each finger offered in (Precup et al., 2020b) and merged in a global nonlinear model of the five fingers as far as the finger angles are concerned. The model-free control system performance is compared with that of the same control system structure, but with PI controllers instead of the iP ones, and the free 
parameters of all controllers are optimally tuned in a model-based manner by a metaheuristic Grey Wolf Optimizer (GWO) algorithm.

This paper is structured as follows. Details on the control system structure and process models are given in the next section. The model-free control tuning approach is formulated in Section 3. The simulation results and their comparison are presented in Section 4. The conclusions are highlighted in Section 5.

\section{Control System Structure and Process Models}

The placement of myoelectric sensors on the human hand and hardware details on the experimental setup are given in (Precup et al., 2018; Teban et al., 2018). The placement of the eight sensors is (Teban et al., 2018): sensors 1 to 4 on the flexor digitorum superficialis, sensors 5,6 on the extensor digitorum, sensor 7 on the extensor digiti minimi, and sensor 8 on the abductor pollicis longus. The placement of all eight sensors on second author's hand is illustrated in (Precup et al., 2020b).

The outputs of all fuzzy models are the angles $y_{l, k}(\%)$ of the fingers $l, l=1 \ldots 5$, expressed as flexion percentages of finger closing between fully relaxed ( 0 , corresponding to minimum contraction) and fully contracted (100, corresponding to maximum contraction), which are subjected to the constraints (Precup et al., 2020b)

$$
0 \% \leq y_{l, k} \% \leq 100 \%, l=1 \ldots 5
$$

with the following finger indices $l: l=1$ (the thumb), $l=2$ (the index finger), $l=3$ (the middle finger), $l=4$ (the ring finger), and $l=5$ (the pinky).

As pointed out in (Precup et al., 2020b), the finger angles (which are actually the finger flexion angles) are measured using five flex sensors; the sensor output is converted to a percentage from $0 \%$ to $100 \%$, where $0 \%$ represents the finger in strait position and $100 \%$ represents the finger in fully bent position. The minimum and maximum bend of each finger is calibrated at startup.

The general expressions of the fuzzy models are (Precup et al., 2020b)

$y_{l, k}=f_{l}\left(\mathbf{z}_{k}\right), \quad l=1 \ldots .5$, where $f_{l}$ are nonlinear input-output maps, $k$ is the discrete time index, and $\mathbf{z}_{k}$ are the input vectors. The elements of vectors $\mathbf{z}_{k}$ are past process outputs and/or inputs; since the fuzzy models are nonlinear, the fuzzy models briefly described in (2) are nonlinear autoregressive with exogenous inputs (NARX) models. The index $k$ will be added as follows as an additional subscript to certain variables that are involved in the controller design because the discrete-time design and implementation of the controllers are carried out.

The internal structure of fuzzy models uses the fuzzification module with Gaussian input membership functions $\mu_{i j}, i=1 \ldots n_{R}\left(n_{R}-\right.$ the number of rules) $j=1 \ldots n$ ( $n-$ the number of input variables) of fuzzy sets of input linguistic terms $L T_{i j}$

$\mu_{i j}\left(z_{j}\right)=\exp \left[-4\left(z_{j}-z_{i j}^{*}\right)^{2} / r_{s}^{2}\right]$

$i=1 \ldots n_{R}, j=1 \ldots n$,

where $z_{j}, j=1 \ldots n$, are the input and also scheduling variables, $n$ is the number of input variables, and $z_{i j}^{*}, i=1 \ldots n_{R}, j=1 \ldots n$, are the membership function centres.

The expression of the rule base of fuzzy models, specific to Takagi-Sugeno-Kang ones, is

Rule $i$ : IF $z_{1} \operatorname{IS} L T_{i 1}$ AND $\ldots$ AND $z_{n}$ IS $L T_{i n}$

THEN $y_{l}^{i}=a_{i 0}+a_{i 1} z_{1}+\ldots+a_{i n} z_{n}, i=1 \ldots n_{R}$,

where $y_{l}^{i}$ is the output of $i^{\text {th }}$ local model in the rule consequent of rule $i, i=1 \ldots n_{R}$, and $a_{i \chi}, i=1 \ldots n_{R}, \chi=0 \ldots n$, are the parameters in the rule consequents.

The fuzzy model structure uses the algebraic product t-norm as an AND operator in the inference engine and the weighted average defuzzification method. Therefore, the expression of the fuzzy model output $y_{l}$ is

$$
\begin{gathered}
y_{l}=\frac{\sum_{i=1}^{n_{R}} \tau_{i} y_{l}^{i}}{\sum_{i=1}^{n_{R}} \tau_{i}}=\sum_{i=1}^{n_{R}} \lambda_{i} y_{l}^{i}, \\
\lambda_{i}=\frac{\tau_{i}}{\sum_{i=1}^{n_{R}} \tau_{i}}, i=1 \ldots n_{R},
\end{gathered}
$$

where all variables depend on $\mathbf{z}$, and $\tau_{i}$ is the firing degree of rule $i$. Additional details are offered in (Precup et al., 2020b). 
The input vector of the best fuzzy model for the first finger $(l=1)$ is (Precup et al., 2020b)

$\mathbf{z}_{k}=\left[z_{1, k} z_{2, k} z_{3, k} z_{4, k} z_{5, k} z_{6, k} z_{7, k} z_{8, k}\right.$

$z_{1, k-1} z_{2, k-1} z_{3, k-1} z_{4, k-1} z_{5, k-1} z_{6, k-1} z_{7, k-1}$

$\left.\begin{array}{lllllll}z_{8, k-1} & y_{1, k-1} & y_{2, k-1} & y_{3, k-1} & y_{4, k-1} & y_{5, k-1} & y_{1, k-2}\end{array}\right]^{T}$,

it evolved to $n_{R}=19$ rules, the number of evolved parameters is 1273 , and its structure and parameter values are available, for full transparency, in the fis file given in (Precup et al., 2020c).

The input vector of the best fuzzy model for the second finger $(l=2)$ is (Precup et al., 2020b)

$\mathbf{z}_{k}=\left[z_{1, k} z_{2, k} z_{3, k} z_{4, k} z_{5, k} z_{6, k} z_{7, k} z_{8, k}\right.$

$\left.\begin{array}{llllll}y_{1, k-1} & y_{2, k-1} & y_{3, k-1} & y_{4, k-1} & y_{5, k-1} & y_{2, k-2}\end{array}\right]^{T}$,

it evolved to $n_{R}=24$ rules, the number of evolved parameters is 1032, and its structure and parameter values are available in the fis file given in (Precup et al., 2020c).

The input vector of the best fuzzy model for the third finger $(l=3)$ is (Precup et al., 2020b)

$\mathbf{z}_{k}=\left[z_{1, k} z_{2, k} z_{3, k} z_{4, k} z_{5, k} z_{6, k} z_{7, k} z_{8, k}\right.$

$z_{1, k-1} z_{2, k-1} z_{3, k-1} z_{4, k-1} z_{5, k-1} z_{6, k-1} z_{7, k-1}$

$\left.\begin{array}{lllllll}z_{8, k-1} & y_{1, k-1} & y_{2, k-1} & y_{3, k-1} & y_{4, k-1} & y_{5, k-1} & y_{3, k-2}\end{array}\right]^{T}$,

it evolved to $n_{R}=27$ rules, the number of evolved parameters is 1809 , and its structure and parameter values are available in the fis file given in (Precup et al., 2020c).

The input vector of the best fuzzy model for the fourth finger $(l=4)$ is (Precup et al., 2020b)

$$
\begin{aligned}
& \mathbf{z}_{k}=\left[z_{1, k} z_{2, k} z_{3, k} z_{4, k} z_{5, k} z_{6, k} z_{7, k} z_{8, k}\right. \\
& \left.\begin{array}{llllll}
y_{1, k-1} & y_{2, k-1} & y_{3, k-1} & y_{4, k-1} & y_{5, k-1} & y_{4, k-2}
\end{array}\right]^{T} \text {, }
\end{aligned}
$$

it evolved to $n_{R}=35$ rules, the number of evolved parameters is 1505 , and its structure and parameter values are available in the fis file given in (Precup et al., 2020c).

The input vector of the best fuzzy model for the fifth finger ( $l=5)$ is (Precup et al., 2020b)

$$
\begin{aligned}
& \mathbf{z}_{k}=\left[z_{1, k} z_{2, k} z_{3, k} z_{4, k} z_{5, k} z_{6, k} z_{7, k} z_{8, k}\right. \\
& z_{1, k-1} z_{2, k-1} z_{3, k-1} z_{4, k-1} z_{5, k-1} z_{6, k-1} z_{7, k-1} \\
& \left.z_{8, k-1} y_{1, k-1} y_{2, k-1} y_{3, k-1} y_{4, k-1} y_{5, k-1} y_{5, k-2}\right]^{T} \text {, }
\end{aligned}
$$

it evolved to $n_{R}=23$ rules, the number of evolved parameters is 1541 , and its structure and parameter values are available in the fis file given in (Precup et al., 2020c).

These models have many parameters. However they are accurate, as proved in (Precup et al., $2020 \mathrm{~b}$ ), in describing the nonlinear mechanisms specific to the process. That is the reason why they are used in the simulation of the control system behaviour.

The MIMO control system structure for finger angle control is presented in Figure 1. It consists of five separate control loops that control each finger using the following control signals (Precup et al., 2020b):

$u_{1}=z_{6}, u_{2}=z_{3}, u_{3}=z_{2}, u_{4}=z_{1}, u_{5}=z_{8}$,

where $u_{l}, l=1 \ldots 5$, is the control signal of the control loop that controls the output (namely, finger angle) $y_{l}, l=1 \ldots .5$. The other notations in Figure 1 are $r_{l}, l=1 \ldots 5$ - reference inputs (set-points) for the controlled outputs (namely, finger angles) $y_{l}, l=1 . .5, e_{l}, e_{l}=r_{l}-y_{l}$, $l=1 \ldots 5-$ control errors, $d_{y l}, l=1 \ldots 5$ disturbances, $C_{l}, l=1 . . .5$ - controllers, which are in this paper iP controllers to be designed and tuned in the next two sections and PI controllers to be designed and tuned for comparison in Section 4.

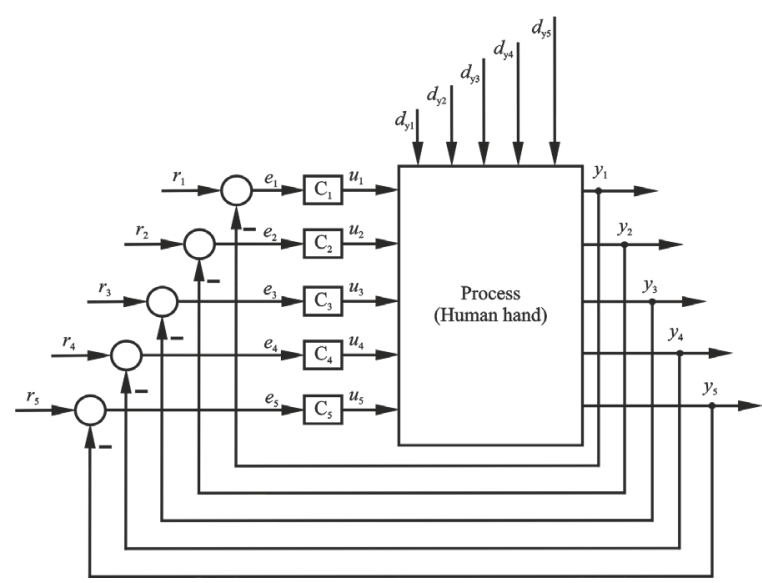

Figure 1. MIMO control system structure for finger angle control (Precup et al., 2020b)

\section{Model-free Control Tuning Approach}

The design of the discrete-time MFC algorithm, further referred as iP controller starts with the first-order local process model presented in 
the Single Input-Single Output (SISO) case in (Join et al., 2020; Roman et al., 2018)

$y_{k+1}=y_{k}+\alpha u_{k}+F_{k}$,

and the MIMO case in (Roman et al., 2016), where $u_{k}$ is the control signal, $y_{k}$ is the controlled output, $\alpha$ is a parameter which guarantees that $\Delta y_{k+1}=y_{k+1}-y_{k}$ and $\alpha u_{k}$ have the same order of magnitude, and $F_{k}$, which accounts for the unmodeled dynamics and disturbances, is built using only the input/output data in the SISO case (Join et al., 2020; Roman et al., 2018) and the MIMO case (Roman et al., 2016).

The discrete-time control law of the MFC algorithms is:

$u_{k}=\alpha^{-1}\left(-\hat{F}_{k}+r_{k+1}-r_{k}-K e_{k}\right)$

where $K$ is the proportional gain associated to the proportional element of the iP controller, $r_{k}$ is the reference input (for example, desired trajectory) that can be considered the output of the refence model, $e_{k}$ is the control error and is expressed as follows (Join et al., 2020; Roman et al., 2018):

$e_{k}=r_{k}-y_{k}$,

$\hat{F}_{k}$ is the estimate of $F_{k}$, computed using the one-step behind and the current input/output measured data of the process, and it is defined as (Join et al., 2020; Roman et al., 2016; Roman et al., 2018):

$\hat{F}_{k}=y_{k}-y_{k-1}-\alpha u_{k-1}$.

Due the fact that it is almost impossible to measure the estimation error, it will be estimated as $\delta_{k}$ using $\hat{F}_{k}$ and $F_{k}$ :

$\delta_{k}=F_{k}-\hat{F}_{k}$.

The dynamics of the closed-loop control system structure is obtained using the first-order local process model in (12) and the control law in (13) in the SISO case (Join et al., 2020; Roman et al., 2018):

$y_{k+1}=y_{k}-\hat{F}_{k}+r_{k+1}-r_{k}-K e_{k}+F_{k}$,

and the MIMO case is treated in (Roman et al., 2016).

The control system structure with MFC algorithm is illustrated in Figure 2.

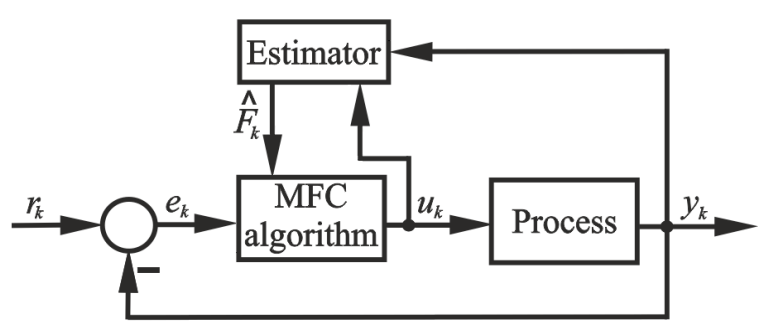

Figure 2. The control system structure with MFC algorithm

In the particular case of the myoelectric-based controlled prosthetic hand process, the controller will consist of five SISO MFC controllers that will run in parallel. Therefore, the expression of the parameter vector of the MFC iP controller is:

$\boldsymbol{\rho}=\left[K_{1} \alpha_{1} K_{2} \alpha_{2} K_{3} \alpha_{3} K_{4} \alpha_{4} K_{5} \alpha_{5}\right]$,

where $K_{1}$ and $\alpha_{1}$ are parameters of the iP controller of the thumb, $K_{2}$ and $\alpha_{2}$ are parameters of the iP controller of the index finger, $K_{3}$ and $\alpha_{3}$ are parameters of the iP controller of the middle finger, $K_{4}$ and $\alpha_{4}$ are parameters of the iP controller of the ring finger, $K_{5}$ and $\alpha_{5}$ are parameters of the iP controller of the pinky.

The design approach of the discrete-time MFC algorithm consists of the following steps:

Step 1. Separate SISO MFC controllers are implemented for each input of the MIMO process to ensure the desired finger dynamics of the control structure.

Step 2. The optimal parameters of the MFC algorithm gathered in vector $\boldsymbol{\rho}$ in (18) are determined using GWO algorithm by solving an optimization problem that will minimize an integral of time-weighted absolute error (ITAE) objective function.

\section{Simulation Results and Comparison}

Two scenarios are proposed to validate the approach. In both scenarios the discrete-time MFC algorithm is validated using simulations on the myoelectric-based controlled prosthetic hand process. Each simulation trial takes $300 \mathrm{~s}$ and the sampling period is set to $T_{s}=0.01 \mathrm{~s}$. The discrete-time MFC system performance is compared with that of the same control system structure, but with PI controllers instead of the 
iP ones, and all the free parameters of both controllers are optimally tuned in a model-based manner by a metaheuristic GWO algorithm solving the following optimization problem:

$\boldsymbol{\rho}^{*}=\arg \min J_{e, u}(\boldsymbol{\rho})$,

$J_{e, u}(\boldsymbol{\rho})=\frac{1}{N}\left(\sum_{k=1}^{N}\left(k\left|e_{1, k}(\boldsymbol{\rho})\right|\right)\right.$

$+\sum_{k=1}^{N}\left(k\left|e_{2, k}(\boldsymbol{\rho})\right|\right)+\sum_{k=1}^{N}\left(k\left|e_{3, k}(\boldsymbol{\rho})\right|\right)$

$\left.+\sum_{k=1}^{N}\left(k\left|e_{4, k}(\mathbf{\rho})\right|\right)+\sum_{k=1}^{N}\left(k\left|e_{5, k}(\mathbf{\rho})\right|\right)\right)$

where $N=30000$ is the number of samples, and $e_{1, k}, e_{2, k}, e_{3, k}, e_{4, k}, e_{5, k}$ are the control errors of the thumb, index, middle, ring and pinky fingers, respectively. The optimization problem in (19) minimizes the ITAE objective function $J_{e, u}(\mathbf{\rho})$. Vector $\rho^{*}$ is the optimal controller parameter vector of the myoelectric-based controlled prosthetic hand process, $\boldsymbol{\rho}$ in (18) is the expression of the tunable parameter vector of the discrete-time iP controller, and

$\boldsymbol{\rho}=\left[K_{1} K_{2} K_{3} K_{4} K_{5} K_{6} K_{7} K_{8} K_{9} K_{10}\right]$

is the expression of the tunable parameter vector of the discrete-time PI controllers with the following transfer functions:

$C_{1}\left(z^{-1}\right)=\frac{K_{1}+K_{2} z^{-1}}{1-z^{-1}}, C_{2}\left(z^{-1}\right)=\frac{K_{3}+K_{4} z^{-1}}{1-z^{-1}}$,

$C_{3}\left(z^{-1}\right)=\frac{K_{5}+K_{6} z^{-1}}{1-z^{-1}}, C_{4}\left(z^{-1}\right)=\frac{K_{7}+K_{8} z^{-1}}{1-z^{-1}}$,

$C_{5}\left(z^{-1}\right)=\frac{K_{9}+K_{10} z^{-1}}{1-z^{-1}}$,

where $C_{1}\left(z^{-1}\right)$ is the transfer function of the discrete-time PI controller for the thumb finger $C_{2}\left(z^{-1}\right)$ is the transfer function of the discretetime PI controller for the index finger, $C_{3}\left(z^{-1}\right)$ is the transfer function of the discrete-time PI controller for the middle finger, $C_{4}\left(z^{-1}\right)$ is the transfer function of the discrete-time PI controller for the ring finger, $C_{5}\left(z^{-1}\right)$ is the discrete-time transfer function of the discretetime PI controller for the pinky, and all transfer functions depend on $\boldsymbol{\rho}$.

The reference trajectory set in both case studies is:

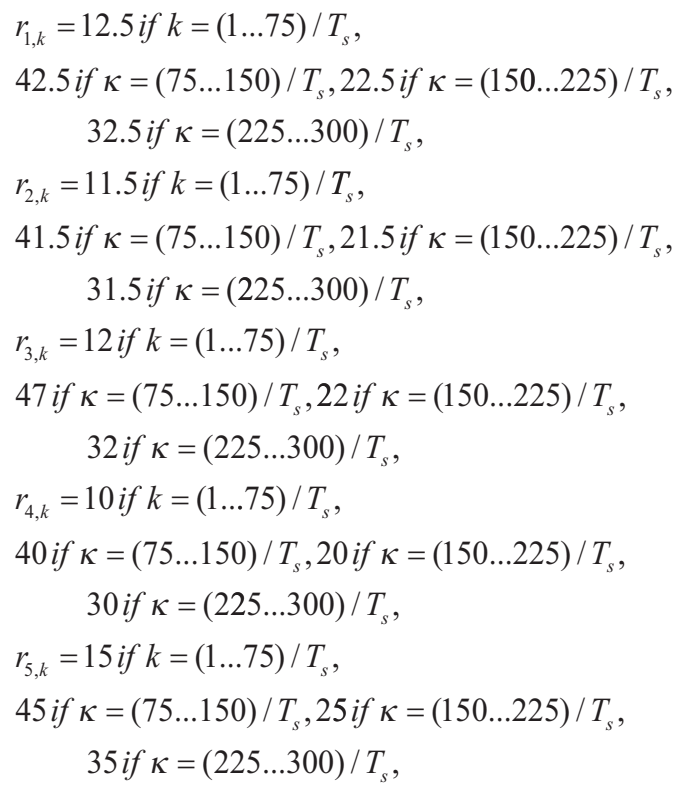

where $r_{1, k}, r_{2, k}, \quad r_{3, k}, r_{4, k}$, and $r_{5, k}$, are the reference trajectories of the thumb, index, middle, ring and pinky fingers, respectively.

The difference between the first and the second scenario is that in the first one no additive disturbances are applied and the following set of additive disturbances is applied directly to the controlled outputs in the second case study to determine how the controller carries out the disturbance rejection:

$d_{y 1, k}=0$ if $\kappa=(0 \ldots 25) / T_{s}$,

5 if $\kappa=(25 \ldots 50) / T_{s},-5$ if $\kappa=(50 \ldots 300) / T_{s}$,

$d_{y 2, k}=0$ if $\kappa=(0 \ldots 30) / T_{s}$,

5 if $\kappa=(30 \ldots 60) / T_{s},-5$ if $\kappa=(60 \ldots 300) / T_{s}$,

$d_{y 3, k}=0$ if $\kappa=(0 \ldots 110) / T_{s}$,

5if $\kappa=(110 \ldots 135) / T_{s},-5$ if $\kappa=(135 \ldots 300) / T_{s}$,

$d_{y 4, k}=0$ if $\kappa=(0 \ldots 190) / T_{s}$,

5 if $\kappa=(190 \ldots 210) / T_{s},-5$ if $\kappa=(210 \ldots 300) / T_{s}$,

$d_{y 5, k}=0$ if $\kappa=(0 \ldots 250) / T_{s}$,

5 if $\kappa=(250 \ldots 285) / T_{s},-5$ if $\kappa=(285 \ldots 300) / T_{s}$,

where $d_{y 1, k} d_{y 2, k}, d_{y 3, k}, d_{y 4, k}$ and $d_{y 5, k}$ are the additive disturbances that are applied to the process outputs, i.e., the thumb, index, middle, ring and pinky finger angles.

In both scenarios disturbances appear because the motions of the myoelectric-based controlled prosthetic hand process are interconnected as it usually happens in MIMO control systems. Moreover, as specified in (Precup et al., 2020b), disturbances are actually applied from the other three inputs (the other muscles) 
out of the total eight ones measured by the myoelectric sensors.

The following parameters of GWO that solves the optimization problem defined in (19) were used in order to achieve an acceptable tradeoff to convergence and computational resources: total number of agents (i.e., grey wolves) $N_{G W O}=20$ and maximum number of iterations $\mu_{\max }=100$. Additional details on GWO are given in (Precup et al., 2016).

The optimal parameter vectors of the MIMO MFC algorithm (i.e., the MIMO iP controller) are determined in zero initial conditions by solving the optimization problem in (19) with $\boldsymbol{\rho}$ in (18) considering the reference trajectory vector in (22) and the fuzzy model of the myoelectricbased controlled prosthetic hand process. The result is:

$\boldsymbol{\rho}^{*}=[0.371-47.799-0.9791 .943-0.271$

$41.475-0.1280 .272-0.11133 .441]$.

The optimal parameter vectors of the discrete-time MIMO PI controller are determined in a similar manner as those of the iP controller, in zero initial conditions by solving the optimization problem in (19) with $\rho$ in (20) considering the reference trajectory vector in (22) and again the fuzzy model of the myoelectric-based controlled prosthetic hand process. The result is:

$\boldsymbol{\rho}^{*}=\left[\begin{array}{llll}0.188-0.180 & 12.341-11.330 & 0.222\end{array}\right.$

$-0.2162 .299-2.2703 .317-3.310]$.

To highlight how the optimal parameters of both discrete-time iP and PI controller were obtained using GWO, besides the final controlled outputs of the control systems with iP and PI controllers, Figure 3 also presents the intermediate outputs obtained before achieving the optimal parameters of iP and PI controllers.

The values of the ITAE performance index of the above control structure are: $J_{e, u}=596.81$ for the optimal iP controller, $J_{e, u}=599.74$ for the intermediate iP controller, $J_{e, u}=530.81$ for the optimal PI controller, $J_{e, u} \stackrel{e, u}{=} 566.20$ for the intermediate PI controller. As highlighted in Figure 3 and these values, a considerable improvement of the final results versus the intermediate ones is obtained.
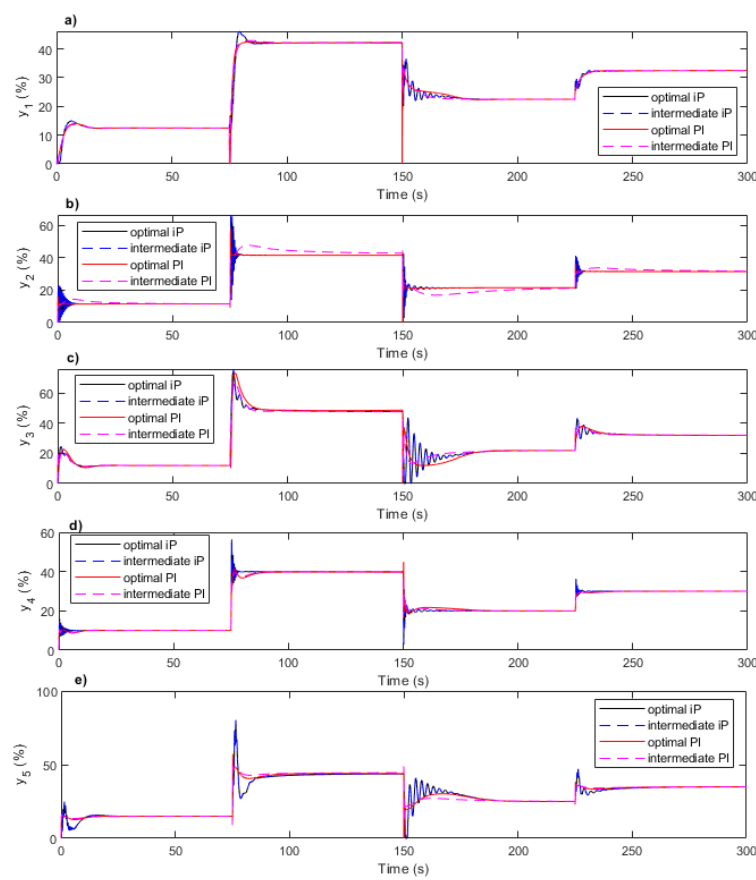

Figure 3. Flexion percentages (finger angles) $y_{1}$ versus time in a), $y_{2}$ versus time in $\mathrm{b}$ ), $y_{3}$ versus time in c), $y_{4}$ versus time in d), $y_{5}$ versus time in e) using optimal iP controller (black), intermediate iP controller (blue), optimal PI controller (red), intermediate PI controller (magenta) in the first scenario

The control signals of the control systems with iP and PI controllers are illustrated in Figure 4.
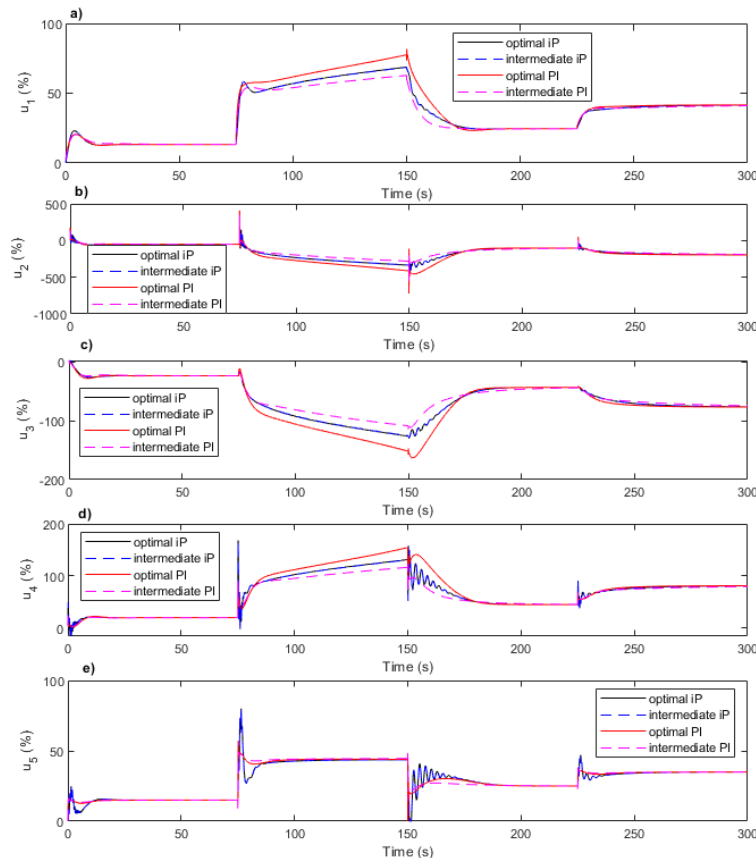

Figure 4. Control signals $u_{1}$ versus time in a), $u_{2}$ versus time in $\mathrm{b}$ ), $u_{3}$ versus time in $\mathrm{c}$ ), $u_{4}$ versus time in d), $u_{5}$ versus time in e) using optimal iP controller (black), intermediate iP controller (blue), optimal PI controller (red), intermediate PI controller (magenta) in the first scenario 
The intermediate results were obtained after 50 iterations of GWO running that led to the following set of parameters:

$\boldsymbol{\rho}=[0.368-47.800-0.9801 .940-0.269$

$41.473-0.1260 .274-0.10833 .440]$

for the iP controller, and

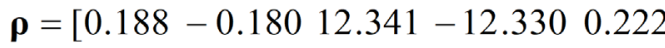

$-0.2162 .299-2.2703 .317-3.310]$

for the PI controller.

The results of the second scenario illustrated in Figures 5 and 6 are obtained using the same optimal and intermediate sets of parameters of the discrete-time iP and PI controllers and applying the additive disturbances in (23) in order to determine how the MIMO controllers manage to reject them.
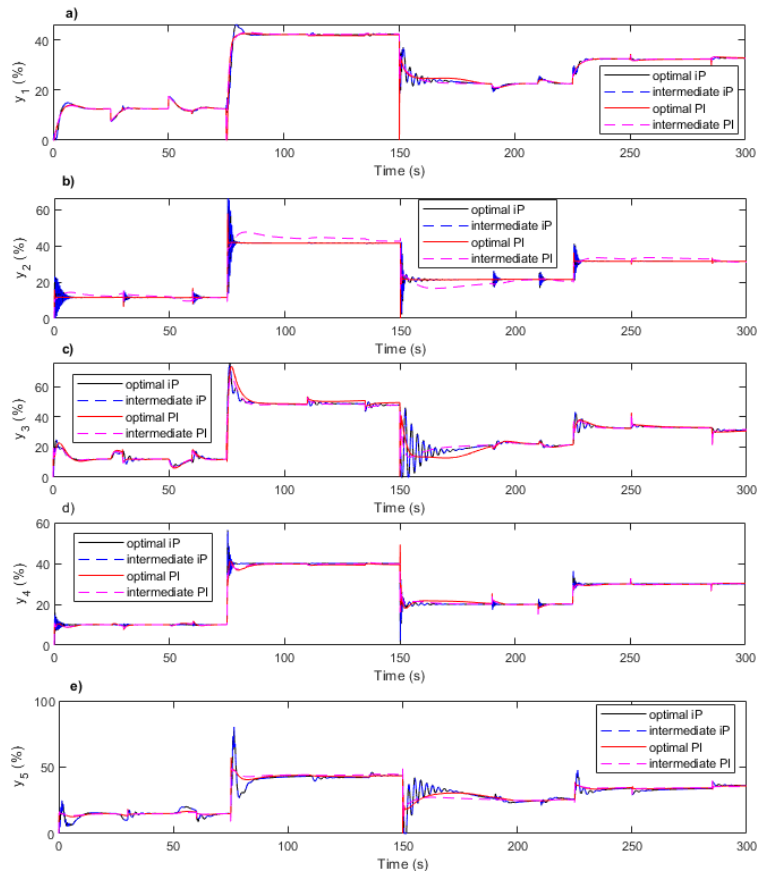

Figure 5. Flexion percentages (finger angles) $y_{1}$ versus time in a), $y_{2}$ versus time in $\mathrm{b}$ ), $y_{3}$ versus time in c), $y_{4}$ versus time in d), $y_{5}$ versus time in e) using optimal iP controller (black), intermediate iP controller (blue), optimal PI controller (red), intermediate PI controller (magenta) in the second scenario

The values of the ITAE performance index in the second scenario are: $J_{e, u}=893.40$ for the optimal iP controller, $J_{e, u}=894.53$ for the intermediate iP controller, $J_{e, u}=876.88$ for the optimal PI controller, $J_{e, u} \stackrel{e, u}{=} 766.00$ for the intermediate PI controller.

As shown in Figures 3 to 6 , the performance of the control structure with iP controller is similar to that of the control structure with PI controller. It is expected that, after applying the proposed controllers to the real-time myoelectric-based controlled prosthetic hand process, the results will be different. This happens because random disturbances can occur at any moment and in this case a better performance will be obtained by the iP controller versus the PI controller. This conclusion is motivated by the fact that the MFC algorithm does not need an accurate model of the process and through it the unexpected disturbances are estimated online.
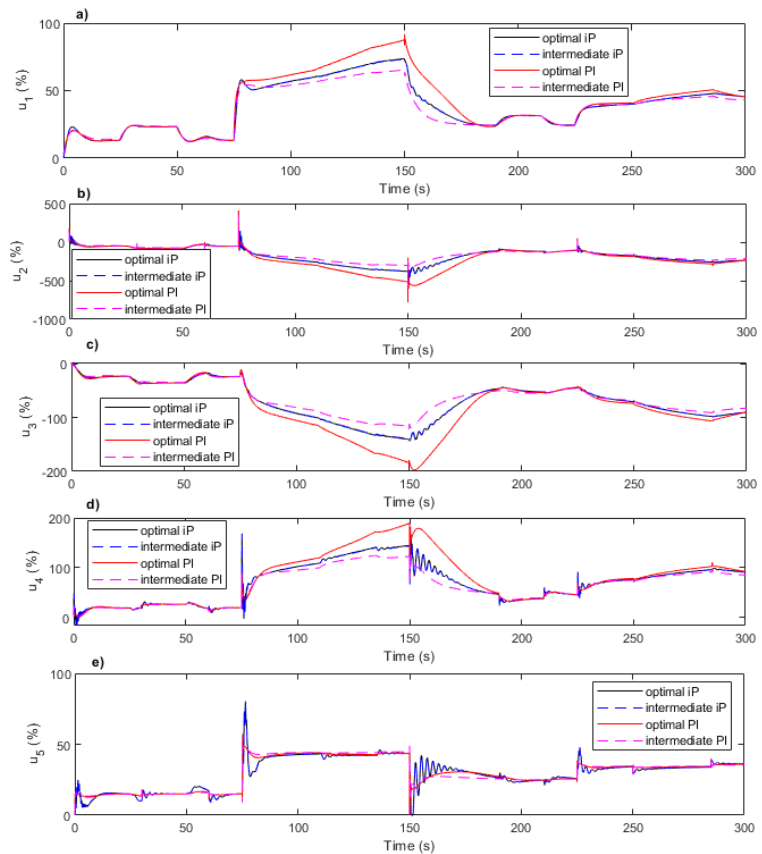

Figure 6. Control signals $u_{1}$ versus time in a), $u_{2}$ versus time in $\mathrm{b}$ ), $u_{3}$ versus time in $\mathrm{c}$ ), $u_{4}$ versus time in d), $u_{5}$ versus time in e) using optimal iP controller (black), intermediate iP controller (blue), optimal PI controller (red), intermediate PI controller (magenta) in the second scenario

However, the conclusions might be slightly different as far as the performance comparison is concerned if other processes are tackled by model-free controllers; such promising examples including the medical ones are fault diagnosis (Baranyi et al., 2004), real-time medical telemonitoring (Costin et al., 2009), telerobotic systems serving space medicine (Haidegger et al., 2011), hydraulic plants (Angelov et al., 2013), microdrilling operations (Beruvides et al., 2013), smart floors (Hvizdoš et al., 2015), chaotic systems (Precup \& Tomescu, 2015), turbojet engines (Andoga \& Fozo, 2017), fuzzy systems (Dzitac et al., 2017) and autonomous systems (Deliparaschos et al., 2020). 


\section{Conclusion}

This paper proposes an MFC iP data-driven algorithm whose parameters were optimally tuned in a model-based manner using the mathematical model of the myoelectric-based controlled prosthetic hand process to control the thumb, index, middle, ring and pinky finger angles. The performance of the MIMO iP controller was compared with that of a MIMO PI controller, where both controllers had the same number of free parameters. It was highlighted that both controller parameters were obtained after solving an optimization problem in terms of metaheuristic GWO.

Future work will focus on validating other nonlinear controllers on the myoelectric-based controlled prosthetic hand process and also on reducing the number of parameters. One solution is to deal with two-degree-of-freedom (2-DOF) fuzzy controllers; the concept of 2-DOF fuzzy

\section{REFERENCES}

Abed-alguni, B. H. (2019). Island-based Cuckoo Search with Highly Disruptive Polynomial Mutation, International Journal of Artificial Intelligence, 17(1), 57-82.

Ameri, A., Akhaee, M. A., Scheme, E. \& Englehart, K. (2019). Regression Convolutional Neural Network for Improved Simultaneous EMG Control, Journal of Neural Engineering, 16(3), 036015.

Andoga, R. \& Fozo, L. (2017). Near Magnetic Field of a Small Turbojet Engine, Acta Physica Polonica A, 131(4), 1117-1119.

Angelov, P., Škrjanc, I. \& Blažič, S. (2013). Robust Evolving Cloud-based Controller for a Hydraulic Plant. In Proceedings of 2013 IEEE Conference on Evolving and Adaptive Intelligent Systems, Singapore (pp. 1-8)

Bara, O., Fliess, M., Join, C., Day, J. \& Djouadi, S. M. (2018). Toward a Model-free Feedback Control Synthesis for Treating Acute Inflammation, Journal of Theoretical Biology, 448, 26-37.

Baranyi, P., Korondi, P., Patton, R. J. \& Hashimoto, H. (2004). Trade-off Between Approximation Accuracy and Complexity for TS Fuzzy Models, Asian Journal of Control, 6(1), 21-33.

Beruvides, G., Quiza, R., Del Toro, R. \& Haber, R. E. (2013). Sensoring Systems and Signal Analysis to Monitor Tool Wear in microdrilling Operations control was coined by Precup \& Preitl (1999) and Precup \& Preitl (2003) as fuzzy control with non-homogenous dynamics with respect to the input channels, and further developed in their later works applied to servo systems and electrical drives (Precup et al., 2009; Preitl et al., 2012). Another solution is to change the optimization problems and algorithms, with useful examples that deal with path planning (Purcaru et al., 2013), fuzzy classification systems (Johanyák, 2017), asymmetric traveling salesman problem (Osaba et al., 2018), benchmark functions (Abed-alguni, 2019), controller tuning (So, 2019), and the application of type-2 fuzzy control (Li et al., 2018; Moreno et al., 2020).

\section{Acknowledgements}

The research reported in this paper was supported by the contract PN-III-P1-1.1-PD-2019-0637 and by the NSERC of Canada.

on a Sintered Tungsten-Copper Composite Material, Sensors and Actuators, A: Physical, 199, 165-175.

Blana, D., van den Bogert, A. J., Murray, W. M., Ganguly, A., Krasoulis, A., Nazarpour, K. \& Chadwick, E. K. (2020). Model-Based Control of Individual Finger Movements for Prosthetic Hand Function, IEEE Transactions on Neural Systems and Rehabilitation Engineering, 28(3), 612-620.

Campi, M. C., Lecchini, A. \& Savaresi, S. M. (2002). Virtual Reference Feedback Tuning: A Direct Method for the Design of Feedback Controllers, Automatica, $38(8), 1337-1346$.

Costin, H., Rotariu, C., Alexa, I., Constantinescu, G., Cehan, V., Dionisie, B., Andruseac, G., Felea, V., Crauciuc, E. \& Scutariu, M. (2009). TELEMON - A Complex System for Real Time Medical Telemonitoring. In Proceedings of $11^{\text {th }}$ International Congress of the IUPESM/World Congress on Medical Physics and Biomedical Engineering, Munich, Germany (pp. 92-95).

da Silva Moreira, J., Acioli Júnior, G. \& Rezende Barros, G. (2018). Time and Frequency Domain Datadriven PID Iterative Tuning, IFAC-PapersOnLine, 51(15), 1056-1061.

Deliparaschos, K. M., Michail, K. \& Zolotas, A. C. (2020). Facilitating Autonomous Systems with AIbased Fault Tolerance and Computational Resource Economy, Electronics, 9(5), 788. 
Dzitac, I., Filip, F. G. \& Manolescu, M.-J. (2017). Fuzzy Logic is Not Fuzzy: World-renowned Computer Scientist Lotfi A. Zadeh, International Journal of Computers Communication and Control, 12(6), 748-789.

Fliess, M. \& Join, C. (2009). Model-free Control and Intelligent PID Controllers: Towards a Possible Trivialization of Nonlinear Control?, IFAC Proceedings Volumes, 42(10), 1531-1550.

Fliess, M. \& Join, C. (2013). Model-free Control, International Journal of Control, 86(12), 2228-2252.

Fliess, M. \& Join, C. (2020). Machine Learning and Control Engineering: The Model-free Case. In Proceedings of Future Technologies Conference 2020, Vancouver, BC, Canada (pp. 1-20).

Formentin, S., Campi, M. C., Caré, A. \& Savaresi, S. M. (2019). Deterministic Continuous-time Virtual Reference Feedback Tuning (VRFT) with Application to PID Design, Systems and Control Letters, 127, 25-34.

Galluppi, O., Formentin, S., Novara, C. \& Savaresi, S. M. (2019). Multivariable D2-IBC and Application to Vehicle Stability Control, ASME Journal of Dynamic Systems, Measurement and Control, 141(10), 1-12.

Gao, Z. (2006). Active Disturbance Rejection Control: A Paradigm Shift in Feedback Control System Design. In Proceedings of 2006 American Control Conference, Minneapolis, MN, USA (pp. 2399-2405).

Geethanjali, P. (2016). Myoelectric Control of Prosthetic Hands: State-of-the-art Review, Medical Devices: Evidence and Research, 9, 247-255.

Haidegger, T., Kovács, L., Precup, R.-E., Preitl, S., Benyó, B. \& Benyó, Z. (2011). Cascade Control for Telerobotic Systems Serving Space Medicine, IFAC Proceedings Volumes, 44(1), 3759-3764.

Halmevaara, K. \& Hyötyniemi, H. (2006). Data-based Parameter Optimization of Dynamic Simulation Models. In Proceedings of $47^{\text {th }}$ Conference on Simulation and Modelling, Helsinki, Finland (pp. 68-73).

Hjalmarsson, H. (2002). Iterative Feedback Tuning An Overview, International Journal of Adaptive Control and Signal Processing, 16(5), 373-395.

Hou, Z.-S. \& Wang, Z. (2013). From Model-based Control to Data-driven Control: Survey, Classification and Perspective, Information Sciences, 235, 3-35.

Huang, J.-W. \& Gao, J.-W. (2020). How Could Data Integrate with Control? A Review on Data-based Control Strategy, International Journal of Dynamics and Control, 8(4), 1-11. DOI: 10.1007/s40435-02000688-x
Hvizdoš, J., Vaščák, J. \& Březina, A. (2015). Object Identification and Localization by Smart Floors. In Proceedings of IEEE $19^{\text {th }}$ International Conference on Intelligent Engineering Systems, Bratislava, Slovakia (pp. 113-117).

Igual, C., Igual, J., Hahne, J. M. \& Parra, L.C. (2019). Adaptive Auto-Regressive Proportional Myoelectric Control, IEEE Transactions on Neural Systems and Rehabilitation Engineering, 27(2), 314-322.

Jiang, P., Cheng, Y.-Q., Wang, X.-N. \& Feng, Z. (2016). Unfalsified Visual Servoing for Simultaneous Object Recognition and Pose Tracking, IEEE Transactions on Cybernetics, 46(12), 3032-3046.

Johanyák, Z. C. (2017). A Modified Particle Swarm Optimization Algorithm for the Optimization of a Fuzzy Classification Subsystem in a Series Hybrid Electric Vehicle, Tehnički Vjesnik - Technical Gazette, 24(2), 295-301.

Join, C., Fliess, M. \& Chaxel, F. (2020). Model-Free Control as a Service in the Industrial Internet of Things: Packet Loss and Latency Issues via Preliminary Experiments. In Proceedings of $28^{\text {th }}$ Mediterranean Conference on Control and Automation, SaintRaphaël, France (pp. 1-6).

Jung, H., Jeon, K., Kang, J.-G. \& Oh, S. (2020). Iterative Feedback Tuning of Cascade Control of Twoinertia System, IEEE Control Systems Letters, 5(3), 785-790.

Kadali, R., Huang, B. \& Rossiter, A. (2003). A Data driven Subspace Approach to Predictive Controller Design, Control Engineering Practice, 11(3), 261-278.

Kammer, L. C., Bitmead, R. R. \& Bartlett, P. L. (2000). Direct Iterative Tuning via Spectral Analysis, Automatica, 36(9), 1301-1307.

Kapelner, T., Vujaklija, I., Jiang, N., Negro, F., Aszmann, O. C., Principe, J. \& Farina, D. (2019). Predicting Wrist Kinematics from Motor Unit Discharge Timings for the Control of Active Prostheses, Journal of NeuroEngineering and Rehabilitation, 16(1), 47.

Karimi, A., Miskovic, L. \& Bonvin, D. (2004). Iterative Correlation-based Controller Tuning, International Journal of Adaptive Control and Signal Processing, 18(8), 645-664.

Li, H.-Y., Wang, J.-H., Wu, L.-G., Lam, H.-K. \& Gao, Y.-B. (2018). Optimal Guaranteed Cost SlidingMode Control of Interval Type-2 Fuzzy Time-Delay Systems, IEEE Transactions on Fuzzy Systems, 26(1), 246-257.

Lucchini, A., Formentin, S., Corno, M., Piga, D. \& Savaresi, S. M. (2020). Torque Vectoring for Highperformance Electric Vehicles: a data-driven MPC Approach, IEEE Control Systems Letters, 4(3), 725-730.

https://www.sic.ici.ro 
McDaid, A. J., Aw, K. C., Haemmerle, E. \& Xie, S. Q. (2012). Control of IPMC Actuators for Microfluidics with Adaptive "Online" Iterative Feedback Tuning, IEEE/ ASME Transactions on Mechatronics, 17(4), 789-797.

Moreno, J. E., Sanchez, M. A., Mendoza, O., Rodríguez Díaz, A., Castillo, O., Melin, P. \& Castro, J. R. (2020). Design of an Interval Type-2 Fuzzy Model with Justifiable Uncertainty, Information Sciences, 513, 206-221.

Novara, C., Formentin, S., Savaresi, S. M. \& Milanese, M. (2015). A Data-driven Approach to Nonlinear Braking Control. In Proceedings of $54^{\text {th }}$ IEEE Conference on Decision and Control, Osaka, Japan (pp. 1-6).

Osaba, E., Del Ser, J., Sadollah, A., Bilbao, M. N. \& Camacho, D. (2018). A Discrete Water Cycle Algorithm for Solving the Symmetric and Asymmetric Traveling Salesman Problem, Applied Soft Computing, $71,277-290$

Precup, R.-E., David, R.-C., Petriu, E.M., SzedlakStinean, A.-I. \& Bojan-Dragos, C.-A. (2016). Grey Wolf Optimizer-based Approach to the Tuning of PIFuzzy Controllers with a Reduced Process Parametric Sensitivity, IFAC-PapersOnLine, 49(5), 55-60, 2016.

Precup, R.-E. \& Preitl, S. (1999). Development of Some Fuzzy Controllers with Non-Homogenous Dynamics with Respect to the Input Channels Meant for a Class of Systems. In Proceedings of 1999 European Control Conference, Karlsruhe, Germany (pp. 61-66).

Precup, R.-E. \& Preitl, S. (2003). Development of Fuzzy Controllers with Non-homogeneous Dynamics for Integral-type Plants, Electrical Engineering, 85(3), 155-168.

Precup, R.-E., Preitl, S., Petriu, E. M., Roman, R.-C., Bojan-Dragos, C.-A., Hedrea, E.-L. \& SzedlakStinean, A.-I. (2020a). A Center Manifold Theorybased Approach to the Stability Analysis of State Feedback Takagi-Sugeno-Kang Fuzzy Control Systems, Facta Universitatis, Series: Mechanical Engineering, 18(2), 189-204.

Precup, R.-E., Preitl, S., Petriu, E. M., Tar, J. K., Tomescu, M. L. \& Pozna, C. (2009). Generic Twodegree-of-freedom Linear and Fuzzy Controllers for Integral Processes, Journal of The Franklin Institute, 346(10), 980-1003.

Precup, R.-E., Teban, T.-A. \& Albu, A. (2019). Evolving Fuzzy and Neural Network Models of Finger Dynamics for Prosthetic Hand Myoelectricbased Control. In Proceedings of $11^{\text {th }}$ International Conference on Electronics, Computers and Artificial Intelligence, Pitesti, Romania (pp. 1-8).

Precup, R.-E., Teban, T.-A., Albu, A., Borlea, A.-B., Zamfirache, I. A. \& Petriu, E. M. (2020b). Evolving
Fuzzy Models for Prosthetic Hand Myoelectric-based Control, IEEE Transactions on Instrumentation and Measurement, 69(7), 4625-4636.

Precup, R.-E., Teban, T.-A., Albu, A., Borlea, A.-B., Zamfirache, I. A. \& Petriu, E. M. (2020c). Best Evolved Fuzzy Models for Prosthetic Hand MyoelectricBased Control. Available at: <http://www.aut.upt. ro/ rprecup/Fuzzy-models.zip>, last accessed: Mar. 3,2020 .

Precup, R.-E., Teban, T.-A., Albu, A., Szedlak-Stinean, A.-I. \& Bojan-Dragos, C.-A. (2018). Experiments in Incremental Online Identification of Fuzzy Models of Finger Dynamics, Romanian Journal of Information Science and Technology, 21(4), 358-376.

Precup, R.-E., Teban, T.-A., Petriu, E. M., Albu, A. \& Mituletu, I.-C. (2018). Structure and Evolving Fuzzy Models for Prosthetic Hand Myoelectric-based Control Systems. In Proceedings of $26^{\text {th }}$ Mediterranean Conference on Control and Automation, Zadar, Croatia (pp. 625-630).

Precup, R.-E. \& Tomescu, M.-L. (2015). Stable fuzzy logic control of a general class of chaotic systems, Neural Computing and Applications, 26(3), 541-550.

Preitl, S., Stinean, A.-I., Precup, R.-E., Preitl, Z., Petriu, E. M., Dragos, C.-A. \& Radac, M.-B. (2012). Controller Design Methods for Driving Systems Based on Extensions of Symmetrical Optimum Method with DC and BLDC Motor Applications, IFAC Proceedings Volumes, 45(3), 264-269.

Purcaru, C., Precup, R.-E., Iercan, D., Fedorovici, L.-O., David, R.-C. \& Dragan, F. (2013). Optimal Robot Path Planning Using Gravitational Search Algorithm, International Journal of Artificial Intelligence, 10(S13), 1-20.

Roman, R.-C., Precup, R.-E. \& David, R.-C. (2018). Second Order Intelligent Proportional-Integral Fuzzy Control of Twin Rotor Aerodynamic Systems, Procedia Computer Science, 139, 372-380.

Roman, R.-C., Precup, R.-E. \& Petriu, E. M. (2020). Hybrid Data-driven Fuzzy Active Disturbance Rejection Control for Tower Crane Systems, European Journal of Control. Available at: $<$ https://www.sciencedirect.com/science/article/ abs/pii/S0947358020301667>. DOI: 10.1016/j. ejcon.2020.08.001

Roman, R.-C., Radac, M.-B. \& Precup, R.-E. (2016). Multi-Input-Multi-Output System Experimental Validation of Model-free Control and Virtual Reference Feedback Tuning Techniques, IET Control Theory \& Applications, 10(2), 1395-1403.

Safonov, M. G. \& Tsao, T.-C. (1997). The Unfalsified Control Concept and Learning, IEEE Transactions on Automatic Control, 42(6), 843-847. 
Sartori, M., Durandau, G., Došen, S. \& Farina, D. (2018). Robust Simultaneous Myoelectric Control of Multiple Degrees of Freedom in Wrist-hand Prostheses by Real-Time Neuromusculoskeletal Modeling, Journal of Neural Engineering, 15(6), 066026.

Sato, T., Kusakabe, T., Himi, K., Arakim N. \& Konishi, Y. (2020). Ripple-free Data-driven Dualrate Controller Using Lifting Technique: Application to a Physical Rotation System, IEEE Transactions on Control Systems Technology, PP(99), 1-8. DOI: 10.1109/TCST.2020.2988613

So, G.-B. (2019). EA-Based Design of a Nonlinear PID Controller Using an Error Scaling Technique, Studies in Informatics and Control, 28(3), 279-288. DOI: $10.24846 / v 28 i 3 y 201904$

Spall, J. C. \& Cristion, J. A. (1998). Model-free Control of Nonlinear Stochastic Systems with Discrete-time Measurements, IEEE Transactions on Automatic Control, 43(9), 1198-1210.

Tabakov, M., Fonal, K., Abd-Alhameed, R. A. \& Qahwaji, R. (2016). Fuzzy Bionic Hand Control in Real-time Based on Electromyography Signal Analysis. In Nguyen, N. T., Iliadis, L., Manolopoulos, Y. \& Trawiński, B. (eds.), Computational Collective Intelligence ICCCI 2016, 292-302. Springer, Cham.

Tabakov, M., Fonal, K., Abd-Alhameed, R. A. \& Qahwaji, R. (2018). Bionic Hand Control in Real-time
Based on Electromyography Signal Analysis. In Nguyen, N.T. \& Kowalczyk, R. (eds.), Transactions on Computational Collective Intelligence, XXIX, 21-38. Springer, Cham.

Teban, T.-A., Precup, R.-E., Lunca, E.-C., Albu, A., Bojan-Dragos, C.-A. \& Petriu, E. M. (2018). Recurrent Neural Network Models for Myoelectric-based Control of a Prosthetic Hand. In Proceedings of $22^{\text {nd }}$ International Conference on Systems Theory, Control and Computing, Sinaia, Romania (pp. 603-608).

Van Waarde, H. J., Eising, J., Trentelman, H. L. \& Camlibel, M. K. (2020). Data Informativity: A New Perspective on Data-driven Analysis and Control, IEEE Transactions on Automatic Control, 65(11), 4753-4768. DOI: 10.1109/TAC.2020.2966717

Wang, H.-P., Xu, H., Tian, Y. \& Tang, H. (2020). $\alpha$-Variable Adaptive Model Free Control of iReHave Upper-limb Exoskeleton, Advances in Engineering Software, 148, 102872.

Yu, W., Wang, R., Bu, X.-H. \& Hou, Z.-S. (2020). Model Free Adaptive Control for a Class of Nonlinear Systems with Fading Measurements, Journal of The Franklin Institute, 357(12), 7743-7760.

Zamanipour, M. (2020). A Novelty in Blahut-Arimoto Type Algorithms: Optimal Control Over Noisy Communication Channels, IEEE Transactions on Vehicular Technology, 69(6), 6348-6358. 